\title{
Two Cases of Narcoleptic Patients with Sleep Paralysis as a Chief Complaint
}

\author{
Yong Won Choi, $\mathrm{MD}^{1,2}$, Ji Hyun Song ${ }^{1}$, Tae Won Kim, MD ${ }^{1,2}$, Sung Min Kim, MD ${ }^{1,2}$, \\ In Hee Cho, MD ${ }^{1,2}$, Seung Chul Hong, MD, $\mathrm{PhD}^{1,2}$ \\ 'Sleep Disorders Clinic, Department of Psychiatry, St. Vincent's Hospital, College of Medicine, The Catholic University of Korea, Suwon, Korea \\ ${ }^{2}$ Department of Psychiatry, St. Vincent's Hospital, College of Medicine, The Catholic University of Korea, Suwon, Korea
}

Received: December 4, 2018 Revised: December 20, 2018 Accepted: December 26, 2018 Correspondence Seung Chul Hong, MD, PhD Department of Psychiatry,

St. Vincent's Hospital, College of Medicine,

The Catholic University of Korea,

93 Jungbu-daero, Paldal-gu,

Suwon 16247, Korea

Tel +82-31-249-7114

Fax +82-31-248-6758

E-mail hscjohn@hotmail.com

ORCID

Yong Won Choi

https://orcid.org/0000-0001-7952-2618

Ji Hyun Song

https://orcid.org/0000-0002-0128-2869

Tae Won Kim

https://orcid.org/0000-0003-2689-202X

Sung Min Kim

https://orcid.org/0000-0002-3082-4014

In Hee Cho

https://orcid.org/0000-0003-0694-7249

Seung-Chul Hong

https://orcid.org/0000-0003-0828-2906
Sleep paralysis is considered as a dissociated state during which rapid eye movement sleep related muscle atonia appears while a subject is fully awake. We present a 22 -year-old man and a 19-yearold man diagnosed with narcolepsy who previously visited our clinic with phenomenon of sleep paralysis. When a patient is brought to the clinic with sleep paralysis, various physiologic and medical conditions such as stress, trauma, hereditary factors, physical health, sleep disorders and other psychiatric disorders are taken into consideration. Prevalence of sleep paralysis in narcolepsy patients is known to be $20-50 \%$. Therefore, it is necessary that clinicians should carefully examine the presence or absence of narcoleptic symptoms when dealing with patients with sleep paralysis and should conduct the multiple sleep latency tests confirm the diagnosis of narcolepsy if necessary.

Sleep Med Res 2018;9(2):128-130

Key Words Narcolepsy, Sleep paralysis, Polysomnography, Multiple sleep latency tests.

\section{INTRODUCTION}

Sleep paralysis is a state of inhibited voluntary muscle movements arising at either sleep onset or upon awakening [1]. Most people tend to feel unpleasant while experiencing muscle atonia and they may try to seek explanations for unusual symptoms. Not surprisingly, patients would search for use other nonmedical explanations to make sense of it [2]. A lifetime prevalence rates of sleep paralysis in the general population is estimated to be approximately $8 \%$, though it significantly varies from $2 \%$ to $60 \%$ depending on the study [1]. Besides, higher lifetime prevalence rates were found in students (28.3\%) and in psychiatric patients (31.9\%) [2]. The known causes of sleep paralysis are related to a variety of factors and medical conditions [3]. It is therefore critical for clinicians to understand medical conditions that present sleep paralysis. Thus, we describe two cases of narcolepsy patients who were initially presented to the clinic with sleep paralysis as a chief complaint.

\section{CASE REPORT}

\section{Case 1}

A 22-year-old man was referred to our clinic for an episode of sleep paralysis. The episode of limb muscle weakness occurred when he fell asleep and disappeared spontaneously after 1 to 2 minutes. He showed a regular sleep-wake cycle and neurologic examination was unremarkable. The patient had gastroesophageal reflux disease, but he was not taking any medication. He experienced a feeling of immobility when he was going to sleep, which occurred 3 to 
4 times a week. These episodes were frequently associated with hypnagogic hallucinatory experiences as if something inside of him was expelled from his body. When we carried out a detailed interview, we found out that he had been also experiencing excessive daytime sleepiness. Even though he fell asleep spontaneously while working in the daytime, he had never experienced a sudden and transient episode of muscle weakness accompanied by full conscious awareness, typically triggered by emotional excitement. We have conducted polysomnography and multiple sleep latency tests (MSLT) on the patient. In polysomnography, total sleep time of patient was 543.0 minutes, sleep latency was 2.3 minutes, and rapid eye movement (REM) sleep latency was 7.0 minutes. Sleep architecture was shown as the proportion of each sleep stage of total sleep time (N1, 5.9\%; N2, 56.2\%; N3, 21.4\%; REM, 16.6\%). Fragmented nocturnal sleep without apnea or hypopnea was recorded. Neither epileptiform activity nor respiratory disturbances or periodic leg movements was present. Mean sleep latency was 4.3 minutes with 3 sleep onset rapid eye movement periods (SOREMPs) in the MSLT. Considering the clinical presentation showing absence of cataplexy and laboratory findings, the episode described above was assessed as type 2 narcolepsy. The treatment with daily application of venlafaxine $75 \mathrm{mg}$ for suppressing REM sleep was continued to reduce the frequency of sleep paralysis and hypnagogic hallucination. After taking daily venlafaxine, the number of sleep paralysis with hallucination reduced to once a week. Treatment with modafinil and methylpenidate resulted in a slight reduction of Epworth sleepiness score from 18 to 12.

\section{Case 2}

A 19-year-old man requested evaluation for a 4-year history of sleep paralysis, which began while serving in the military. The patient recalled that his father had experiences of daytime sleepiness when he was a child, yet he had never visited hospital for evaluation. Similarly, the patient himself also had experienced daily symptoms of excessive daytime sleepiness ever since he was in childhood. Since he was familiar with excessive tiredness that had been present throughout his life, he did not take it seriously. He was referred to our sleep clinic to evaluate his symptom of sleep paralysis before joining the military. $\mathrm{He}$ had several episodes of immobility along with a sense of ghost presence or pressure felt on the chest and these occurred while he was going to sleep and undergoing transition from a sleep to wakeful state. He also felt as if his soul escaped from his body. Cataplexy occurred several times a day and typically was manifested as weakness in the knees, triggered by laughter in particular. Based on our history taking and thorough medical interviews, he did not appear to have problems related to circadian rhythm. There were no abnormalities on neurologic examination. He was not taking any medication at that time. We conducted polysomnography and MSLT on the patient. In polysomnography, total sleep time of patient was 553.0 minutes, sleep latency was 1.3 minutes, and REM latency was 0.5 minutes. Sleep architecture was shown as the proportion of each sleep stage of total sleep time (N1, 17.6\%; N2, 49.6\%; N3, $16.1 \%$; REM, 16.7\%). He showed high number of awakenings (54). Neither epileptiform activity nor periodic leg movements was present, while apnea-hypopnea index was $2.3 / \mathrm{h}$. Mean sleep latency was 2.1 minutes with 3 SOREMPs in the MSLT. The episode was assessed as type 1 narcolepsy. Daily application of venlafaxine $75 \mathrm{mg}$ improved cataplexy and sleep paralysis. Treatment with modafinil resulted in a reduction of Epworth sleepiness score from 14 to 8 . These medications alleviated excessive daytime sleepiness, sleep paralysis, and cataplexy.

\section{DISCUSSION}

We described two cases of patients whose chief complaint is muscle atonia during sleep. Even though the patients felt uncomfortable in everyday life due to the presence of daytime sleepiness, they regarded such familiar symptom lightly. No remarkable finding was visible in polysomnography, except fragmented nocturnal sleep with reduction of REM sleep. Thus, we conducted MSLT for identifying the diagnosis of narcolepsy. Afterwards, they started to take medications including stimulants and antidepressants. Daily application of venlafaxine improved cataplexy and sleep paralysis. There is no studies which systematically investigate whether a specific medication improves sleep paralysis [1]. Many clinicians do prescribe antidepressant medications to help alleviate both symptoms of cataplexy and sleep paralysis. The hypothesized mechanism of its action is an increase in postural muscle tone during REM sleep [2]. A 22-year-old man experienced sleep paralysis with a feeling as if his soul escaped from his body when he fell asleep. In the second case, a 19-year-old man had an illusory feeling of his body but also had the sense of ghost presence or pressure felt on the chest when he was going to sleep or waking up. Sleep paralysis is frequently associated with a variety of hallucination. One factor is known as Intruder hallucination, which is consists of sensed presence, fear, and auditory and visual hallucination. Another factor is known as Incubus hallucination composed of pressure felt on the chest. Lastly, Vestibular motor hallucination is consisted of the sense of out-of-body experiences [4]. Isolated sleep paralysis (ISP) is defined as the transient inability to move during the transition between sleep and wakefulness in the absence of other clinical features of narcolepsy. ISP episodes tend to occur during awakening from hypnopompic as opposed to narcolepsy-associated paralysis, which is more closely associated with hypnagogic events [5]. Even though the 19-year-old man had experiences of sleep paralysis with hallucinations when waking up, the majority of hallucinations both patients experienced occurred when they were falling asleep. There are many conditions and diseases that patients should be 
screened for when they visit a clinic with a chief complaint of sleep paralysis. A confirmed or unconfirmed history of childhood sexual abuse and threatening/traumatic events were found to be significantly associated with the frequency of sleep paralysis episodes [1]. A familial association and specific polymorphisms of the PER2 gene were reported to be related with sleep paralysis [1]. Psychiatric disorders including anxiety-related symptoms, post-traumatic stress disorder, panic disorder [6] appear to be associated with sleep paralysis. Sleep paralysis is related to many other sleep related factors. One of the differential diagnoses of presented muscle atonia includes REM sleep behavior disorder. REM sleep behavior disorder is a parasomnia characterized by often frightening dreams related with simple or complex motor behavior and vocalizations during REM sleep [7]. Excessively short $(<6 \mathrm{~h})$ or long $(>9$ h) sleep duration, napping, nightmares appears to be common in sleep paralysis [1]. Shift-work related to sleep disruption was associated with a higher prevalence of sleep paralysis [1]. Besides there is a report that a SOREMPs triggered by sleep disruption led to sleep paralysis [1]. One study showed that sleep paralysis was associated with the use of anxilytic medication (odds ratio = 4.91), but other studies did not demonstrate this correlation [1]. The use of anti-depressants and hypnotic medications was not related to sleep paralysis [8]. Narcolepsy is characterized by excessive daytime sleepiness, cataplexy and sleep paralysis and hypnagogic hallucination and disturbed nocturnal sleep [9]. Orexin suppresses REM sleep, and reduced orexin signaling in narcolepsy may cause elements of REM sleep such as paralysis or dreamlike hallucination to occur during wakefulness [10]. Prevalence of sleep paralysis in patients with narcolepsy is $58.5 \%$ and that of sleep paralysis in controls is $15.1 \%$ [11]. When a patient visits the clinic for sleep paralysis, various conditions and disease such as stress and trauma, hereditary factors, physical health, sleep disorders and other psychiatric disorders should be considered [1]. Clinicians should recognize whether narcoleptic symptoms are present in patients with sleep paralysis, and should be able to conduct the MSLT to confirm the diagnosis of narcolepsy if necessary.

\section{Conflicts of Interest}

The authors have no financial conflicts of interest.

\section{Authors' Contribution}

Conceptualization: Hong S. Data curation: Hong S. Formal analysis: Hong S. Project administration: Hong S. Resources: Hong S. Supervision: Hong S. Validation: Hong S. Writing-original draft: Choi Y. Writingreview \& editing: Hong S, Song J, Kim T, Kim S, Cho I.

\section{REFERENCES}

1. Denis D, French CC, Gregory AM. A systematic review of variables associated with sleep paralysis. Sleep Med Rev 2018;38:141-57.

2. Sharpless BA. A clinician's guide to recurrent isolated sleep paralysis. Neuropsychiatr Dis Treat 2016;12:1761-7.

3. Denis D, French CC, Rowe R, Zavos HM, Nolan PM, Parsons MJ, et al. A twin and molecular genetics study of sleep paralysis and associated factors. J Sleep Res 2015;24:438-46.

4. Cheyne JA, Rueffer SD, Newby-Clark IR. Hypnagogic and hypnopompic hallucinations during sleep paralysis: neurological and cultural construction of the night-mare. Conscious Cogn 1999;8:319-37.

5. McCarty DE, Chesson AL Jr. A case of sleep paralysis with hypnopompic hallucinations. Recurrent isolated sleep paralysis associated with hypnopompic hallucinations, precipitated by behaviorally induced insufficient sleep syndrome. J Clin Sleep Med 2009;5:83-4.

6. Bell CC, Hildreth CJ, Jenkins EJ, Carter C. The relationship of isolated sleep paralysis and panic disorder to hypertension. J Natl Med Assoc 1988;80:289-94

7. Bušková J, Piško J, Dostálová S, Rưžička E, Sonka K. Incomplete sleep paralysis as the first symptom of narcolepsy. Sleep Med 2013;14:91921.

8. Ohayon MM, Zulley J, Guilleminault C, Smirne S. Prevalence and pathologic associations of sleep paralysis in the general population. $\mathrm{Neu}$ rology 1999;52:1194-200.

9. Kallweit U, Bassetti CL. Pharmacological management of narcolepsy with and without cataplexy. Expert Opin Pharmacother 2017;18:80917.

10. Scammell TE. Narcolepsy. N Engl J Med 2015;373:2654-62.

11. Dodet P, Chavez M, Leu-Semenescu S, Golmard JL, Arnulf I. Lucid dreaming in narcolepsy. Sleep 2015;38:487-97. 